\title{
STUDY ON THE DEPENDENCY OF STEADY STATE RESPONSE ON THE RATIO OF LARMOR AND RABI FREQUENCY FOR ESR USING MATLAB
}

\author{
Amit Kumar ${ }^{1}$, Rajib Chakraborty ${ }^{2}$ \\ ${ }^{I}$ Student, Electrical Engineering, Abacus Institute of Engineering and Management, West Bengal, India \\ ${ }^{2}$ Assistant Professor, Physics, Abacus Institute of Engineering and Management, West Bengal, India
}

\begin{abstract}
In this project we simulate with very high accuracy specially to study the dependency of the steady state power and dispersion output on the ratio $(r)$ between Larmor and Rabi frequency for the electron spin resonance experiment by the matlab software (version 7.9.0.529(R2009b)). Where the sample material $(D P P H)$ has been kept in a strong static magnetic field $\left(\boldsymbol{B}_{0}\right)$ and in orthogonal direction a high frequency electromagnetic field $\left(B_{I}(t)\right)$ has been applied. We divide our simulation into two parts. In the first part we ignore the $\omega_{1}^{2} T_{1} T_{2}$ terms and observe the dependency of the power maximum on the amplitude of the oscillating e.m. field $B_{1}$ (for fixed $\left(\omega_{L}\right)$ Larmor frequency) and on $\omega_{L}$ (for fixed $\left.B_{I}\right)$. Also observe a clear shift $(\Delta \omega)$ of the power maxima $\left(P_{\text {max }}\right)$ from $\omega_{L}$. In our second part we consider the $\omega_{1}{ }^{2} T_{1} T_{2}$ term and the ratio $(r)$ between Larmor and Rabi frequency and observe the shift $(\Delta \omega)$ of the power maxima $\left(P_{\max }\right)$ from $\omega_{L}$ and change in peak to peak line width $\left(\Delta B_{P P}\right)$ with $B_{1}$ both depends upon the ratio $r$. we consider various range of $r([0.83,5],[16,100],[88.3,500]$, [1000,2000], [833.3,5000]) and observe these dependency. We observe as the ratio of $r$ increases the output i.e. shift $(\Delta \omega)$ and the change in $\triangle B_{P P}$ with $B_{1}$ decreases and converges to the case of neglecting $\omega_{1}^{2} T_{1} T_{2}$ terms. We also observe the shift $(\Delta \omega)$ follows some non linear relationship with $B_{1}$.
\end{abstract}

Keywords: E.S.R., Larmor, Rabi, Ratio r, Spin.

\section{INTRODUCTION}

In this project we study about the nature of the power absorption and dispersion curve of electron spin resonance. We simulate with very high accuracy specially to study the dependency of the steady state power and dispersion output on the ratio (r) between Larmor and Rabi frequency for the electron spin resonance experiment by the matlab software. Electron paramagnetic resonance (EPR) or electron spin resonance (ESR) is a spectroscopic technique for studying mainly paramagnetic materials having unpaired electrons. The basic concepts of EPR are analogous to those of nuclear magnetic resonance (NMR), where in NMR spins of atomic nuclei are excited but in ESR instead of nuclei the electron spins are excited. EPR spectroscopy is particularly useful for studying metal complexes or organic radicals. In Kazan State University first time EPR was observed by Soviet physicist Yevgeny Zavoisky in 1944, and was developed independently at the same time by Brebis Bleaney at the University of Oxford.

\section{MAGNETIC MOMENT}

Form quantum point of view a free electron (not bounded in an atom) due to its own intrinsic spin angular momentum also possesses a Magnetic moment, which is purely a relativistic effect predicted from relativistic quantum mechanics. The operator of this spin magnetic moment is:

$\mu_{s}=g_{s} \frac{e}{2 m_{e}} s$ where, $s=\frac{\hbar}{2} \partial\left(\partial_{1}, \partial_{2}, \partial_{a} \text { are pauli spin operators }\right)^{[25]}$

The component of the spin operator in any direction has an eigen value $m_{s} \hbar$ (where $m_{s}$ is $= \pm 1 / 2$ spin quantum numbers).So the electrons spin magnetic moment (the Eigen value of the component of spin magnetic moment operator in that direction) is:

$$
\begin{aligned}
& \therefore \mu_{g}=g_{s} \frac{e}{2 m_{e}} \frac{1}{2} \hbar=\frac{g_{g}}{2} \frac{e \hbar}{2 m_{e}} \approx \frac{e h}{2 m_{e}}=\mu_{B} \cdot \text { (Bohr megneton) } \\
& \mu_{\mathrm{B}}=9.2849 * 10^{-24} \mathrm{JT}^{-1}
\end{aligned}
$$

\section{ZEEMAN EFFECT FOR ELECTRON SPIN}

Every electron has a magnetic moment and spin quantum number $s=1 / 2$, with magnetic components $m_{s}=+(1 / 2)$ and $m_{s}=-(1 / 2)$. In the presence of an external magnetic field with strength $\mathrm{B}_{0}$, the electron's magnetic moment aligns itself either parallel $m_{s}=-(1 / 2)$ or anti parallel $m_{s}=+(1 / 2)$ to the field, each alignment having a specific energy due to the Zeeman Effect :

$E=m_{g} g_{g} \mu_{B} B_{0}$

Where

- $g_{\mathrm{s}}$ is the electron's so-called g-factor (see also the Landé $g$-factor). $\mathrm{g}_{\mathrm{s}}=2.0023$ for the free electron

- $\mu_{\mathrm{B}}$ is the Bohr magneton.

Therefore, the separation between the lower and the upper state is $\Delta E=g_{s} \mu_{B} B_{0}$ for unpaired free electrons. This equation implies that the splitting of the energy levels is 
directly proportional to the magnetic field's strength, as shown in the diagram below.

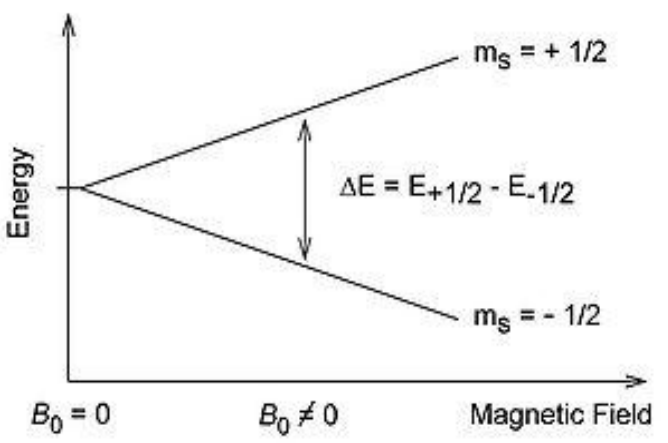

Fig 1: Zeeman Effect

When the resonance happens then an unpaired electron makes a transition between the two energy levels by either absorbing or emitting a photon of energy hv. Then we can write $\hbar \omega=\Delta E$.

\section{LARMOR PRECESSION}

In physics, Larmor precession (named after Joseph Larmor) is the precession of the magnetic moments of electrons, muons, all leptons with magnetic moments, which are quantum effects of particle spin, atomic nuclei, and atoms about an external magnetic field. The magnetic field exerts a torque on the magnetic moment,

$\Gamma=\mu \times B={ }_{J} L \times B$

where $\boldsymbol{\Gamma}$ is the torque, $\boldsymbol{\mu}$ is the magnetic dipole moment, $\mathbf{L}$ is the angular momentum vector, $\boldsymbol{B}$ is the external magnetic field, $X$ symbolizes the cross product, and $\mathrm{y}$ is the gyromagnetic ratio which gives the proportionality constant between the magnetic moment and the angular momentum.

The angular momentum vector $\mathrm{L}$ precesses about the external field axis with an angular frequency known as the Larmor frequency,

$\omega=-y^{B}$

Where $\omega$ is the angular frequency, $y=-\frac{e g}{2 m}$ is (for a particle of charge -e) the gyromagnetic ratio, and B is the magnitude of the magnetic field and $\mathrm{g}$ is the g-factor (normally 1 , except in quantum physics).

Simplified, this becomes:

$$
\left.\omega=\frac{e g B}{2 m} \text { (Energy of the photon } h \omega=q_{\mathrm{e}} \mu_{\mathrm{B}} B_{0}\right)
$$

Where $\omega$ is the Larmor frequency, $m$ is mass, -e is the charge, and B is applied field.

\subsection{Rabi Frequency}

In case of ESR, EPR or NMR Rabi frequency is the nutation frequency of the nutation (a periodic motion superimposed on the precessional circle of the net angular momentum vector) induced due to applied oscillating radio wave , micro wave field $\left(B_{1}(t)\right)$. This is $\omega_{1}=\gamma B_{1}$.

$\left(\mathrm{y}=\right.$ gyromagnetic ratio; $\mathrm{B}_{1}=$ amplitude of the oscillating field)

In quantum mechanical point of view for two level $\left(E_{1} \& E_{2}\right.$; $E_{2}>E_{1}$ ) system the probability of spin flip (i.e. transition from $E_{1}$ to $E_{2}$ level) is a periodic function of time (t);

For any $\omega$ the frequency of the applied oscillating electromagnetic field:

$$
\begin{aligned}
& P_{\text {spin-flip }}(\omega)=\frac{\omega_{1}^{2}}{\Gamma^{2}} \sin ^{2} \frac{\Gamma}{2} t \\
& \omega_{1}=y B_{1} \\
& \Gamma=\sqrt{\Delta^{2}+\omega_{1}^{2}} \\
& \Delta=\omega-\omega_{0} \text { (Detuning factor) }
\end{aligned}
$$

So, angular frequency $\Gamma=\sqrt{\Delta^{2}+\omega_{1}^{2}}$ is called Rabi frequency in quantum mechanics. At resonance when $\omega=\omega_{0}$ then the quantum Rabi frequency became $\Gamma=\sqrt{0+\omega_{1}^{2}}=\omega_{1}=y B_{1}$ (i.e. equal to classical Rabi frequency or nutation frequency). ${ }^{[15,16]}$

\subsection{What is $r$ ?}

$r$ is the ratio of Larmor frequency $\left(\omega_{0}\right.$ or $\left.\omega_{\mathrm{L}}\right)$ and Rabi frequency $\left(\omega_{1}\right)$.

$r=\frac{\omega_{0}}{\omega_{1}}=\frac{\text { Larmor frequency }}{\text { Rabi frequency }}=\frac{\mathrm{y} B_{0}}{\mathrm{y}_{1}}=\frac{B_{0}}{B_{1}}$

\section{MAGNETIC MOMENT DUE TO ELECTRON SPIN IN DPPH}

We know that the electrons spin magnetic moment is

$\therefore \mu_{s}=g_{s} \frac{e}{2 m_{e}} \frac{1}{2} \hat{h}=\frac{g_{s}}{2} \frac{e \hat{h}}{2 m_{e}} \approx \frac{e \hat{h}}{2 m_{e}}=\mu_{B^{*}}$ (Bohr megneton)

$\mu_{\mathrm{B}}=9.2849 * 10^{-24} \mathrm{JT}^{-1}$

Similarly there is nuclear magnetron $\mu_{\mathrm{N}}=5.0571 * 10^{-27} \mathrm{JT}^{-1}$.

It is measured that magnetic moment of the proton

$\mu \mathrm{P}=2.7927 \mu \mathrm{N}$.

$\mu \mathrm{n}=-1.9131 \mu \mathrm{N}$.

$\mu \mathrm{N}=\mathrm{eh} / \mathrm{mp}$

The above numerical value shows that proton and neutron are having magnetic moment $10^{-3}$ time's $\mu_{B} \approx \mu_{e}$.

The nuclei are made up of proton and neutron. The magnetic moments of the nuclei are much smaller than the atomic magnetic moments (generally it is in the order of $\mu_{\mathrm{B}}$.) 
Now we observed for the DPPH (an organic molecule) only one electron attached with the nitrogen atom is unpaired, except this electron all electrons are paired. So we can say the electronic contribution in the molecular magnetic moment of DPPH must be due to this unpaired electron and the nuclear contribution in the molecular magnetic moment caused by all the nucleus (having neutrons and protons) of DPPH molecule must be negligible compared to this unpaired electron. Therefore the net resultant vector of this molecule is obviously in the order of $\mu_{\mathrm{B}}$. It is measured that the molecular $\mathrm{g}$ factor of DPPH is $\mathrm{g}=2.0038$. The $\mathrm{g}$ factor for the electro spin $g_{s}=2.00232$. ${ }^{[9]}$

So we can easily take this molecule as per simulation for electron spin resonance. Affectively for all practical purpose we can consider that the net magnetization vector is due to vector sum of all magnetic dipole of electrons.

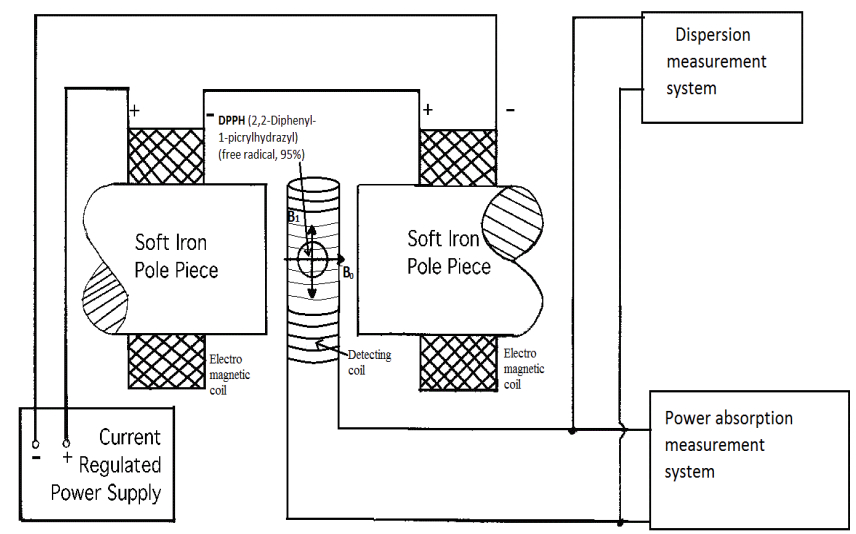

Fig 2: Experimental set up for the project.

\section{FELIX-BLOCH EQUATION}

\section{(Our modeling equation for simulation)}

The magnetization vector $\mathbf{M}$ of DPPH is vector sum of all magnetic dipole moment of electrons in unit volume.

$$
\mathbf{M}=\Sigma_{1} \mu_{1}
$$

(Where $\mathrm{i}=1$ to $\mathrm{N}$; $\mathrm{N}$ is number of electrons in unit volume)

In terms of total angular momentum of a sample

$M=Y * L$

Interaction of magnetic moment with magnetic field gives a torque on the system and changes the angular momentum of the system

$\boldsymbol{\tau}=\frac{d \boldsymbol{L}}{d t}=\boldsymbol{M} \times \boldsymbol{B}$

$\frac{d \boldsymbol{M}}{d t}=y \frac{d \boldsymbol{L}}{d t}=y \boldsymbol{M} \times \boldsymbol{B}$

$\frac{d M}{d t}=y M \times B$
$\boldsymbol{M} \times \boldsymbol{B}=\left|\begin{array}{ccc}i & j & k \\ M_{x} & M_{y} & M_{z} \\ B_{x} & B_{y} & B_{z}\end{array}\right|$

$=\left(M_{y} B_{z}-M_{z} B_{y}\right) i+\left(M_{z} B_{x}-M_{x} B_{z}\right) j+\left(M_{x} B_{y}-M_{y} B_{x}\right) k$

Now electron spins relax to equilibrium value following the application of r.f. fields. Bloch assumed $M_{z}(t)$ relax along $\mathrm{z}$ axis with rate $1 / \mathrm{T} 1$ and $M_{x}(t), M_{y}(t)$ relax in x-y plane with different rate $1 / \mathrm{T} 2$ respectively, $\mathrm{T} 1$ is called spin-lattice relaxation and $\mathrm{T} 2$ is called spin-spin relaxation. Now the Bloch equation with addition of relaxation becomes:

$\frac{d M_{x}(t)}{d t}=\mathrm{Y}(M(t) \times B(t))_{x}-\frac{M_{x}(t)}{T_{2}}$

$\frac{d M_{y}(t)}{d t}=\mathrm{Y}(M(t) \times B(t))_{y}-\frac{M_{y}(t)}{T_{2}}$

$\frac{d M_{z}(t)}{d t}=\mathrm{Y}(M(t) \times B(t))_{z}-\frac{M_{z}(t)-M_{0}}{T_{1}}$

Where $\gamma$ is the gyromagnetic ratio (Taken the value of $\mathrm{g}=2.002322)$

$\mathbf{B}(t)=\left(B_{x}(t), B_{y}(t), B_{z}(\mathrm{t})\right)$ is the applied magnetic field.

$\mathbf{M}(t)=\left(M_{x}(t), \quad M_{y}(t), M_{z}(\mathrm{t})\right)$ three components of magnetization vector.

These set of coupled equations are taken as our modeling equation. The equations are analyzed to simulate the output of E.S.R. experiment. There are three inputs $B_{x}(t), B_{y}(t)$, $B_{z}(\mathrm{t})$ and there are three outputs $M_{x}(t), M_{y}(t), M_{z}(\mathrm{t})$.

\section{STEADY STATE SOLUTION}

In our simulation we have taken inputs as:

$B_{x}(t)=\mathrm{B}_{1} \cos \omega \mathrm{t}$

$B_{y}(t)=-\mathrm{B}_{1} \sin \omega \mathrm{t}$

$B_{z}(\mathrm{t})=\mathrm{B} 0 \quad$ (It is a constant field)

And output is $\mathbf{M}=(\mathbf{M x}, \mathbf{M y}, \mathbf{M z})$. At steady state it is taken as:

$\frac{d u}{d t}=\frac{d v}{d t}=\frac{d M z}{d t}=0$
$M x=u \cos \omega t-v \sin \omega t$
$M y=-u \sin \omega t-v \cos \omega t$

Its matrix representation is given below.

$\left(\begin{array}{l}M x \\ M y\end{array}\right)=\left(\begin{array}{cc}\cos \omega t & -\sin \omega t \\ -\sin \omega t & -\cos \omega t\end{array}\right)\left(\begin{array}{l}u \\ v\end{array}\right)$

In the above equations,

$u=\frac{X_{0} \omega_{0} B_{1}\left(\omega_{0}-\omega\right) T_{2}^{2}}{1+\left(\omega_{0}-\omega\right)^{2} T_{2}^{2}+\omega_{1}^{2} T_{1} T_{2}}$

$\nu=\frac{X_{0} \omega_{0} B_{1} T_{2}}{1+\left(\omega_{0}-\omega\right)^{2} T_{2}^{2}+\omega_{1}^{2} T_{1} T_{2}}$

Complex form of input is given by,

$\boldsymbol{B}=\left(B_{x}+j B_{y}\right)=B_{1} e^{-j \omega t}$

Complex form of magnetization is given by,

$\boldsymbol{M}=M_{x}+j M_{y}=(u-j v)(\cos \omega t-j \sin \omega t)=(u-j v) e^{-j \omega t}$

Now, we can write susceptibility of the system is given by:- 
$X=\frac{M}{B}=\frac{(u-j v) e^{-j \omega t}}{B_{1} e^{-j \omega t}}=\frac{u}{B_{1}}-j \frac{v}{B_{1}}$

Energy stored in the specimen at any instant $(\mathrm{t})$ is given by,

$$
\begin{gathered}
\mathrm{U}=-\mathbf{M} . \mathbf{B} \\
U=-\left(M_{x} B_{x}+M_{y} B_{y}+M_{z} B_{z}\right) \\
U=-\left(M_{x}(t) B_{x}(t)+M_{y}(t) B_{y}(t)+f(\omega) M_{0} B_{0}\right)
\end{gathered}
$$

Power absorbed is, $P=\left\langle\left(\frac{d U}{d t}\right)=\left\langle\left(\frac{d(- \text { M.B })}{d t}\right\rangle\right.\right.$

$P=\left\langle\frac{d\left(-\left(M_{x} B_{x}+M_{y} B_{y}+M_{z} B_{z}\right)\right)}{d t}\right\rangle$

In the above equation value of $M_{z} B_{z}$ is constant. So,

$-P=\left\langle\frac{d U}{d t}\right\rangle=M_{x}(t) \frac{d B_{x}(t)}{d t}+B_{x}(t) \frac{d M_{x}(t)}{d t}+M_{y}(t) \frac{d B_{y}(t)}{d t}+B_{y}(t) \frac{d M_{y}(t)}{d t}$

$\langle P\rangle=2 v \omega B_{1}\left\langle\sin ^{2} \omega t\right\rangle$

$\langle P\rangle=v \omega B_{1}$

\subsection{Output Measurement}

For power output $\mathrm{v}(\omega)$ part is measured and for dispersion output $u(\omega)$ is measured, with the setup shown in figure

So equations for simulations are given below,

For power equation is,

$$
\langle P\rangle=\frac{X_{0} \omega_{0} B_{1} T_{2}}{1+\left(\omega_{0}-\omega\right)^{2} T_{2}^{2}+\omega_{1}^{2} T_{1} T_{2}} \omega B_{1}
$$

And for dispersion we have taken the value of $u(\omega)$.

$$
u=\frac{X_{0} \omega_{0} B_{1}\left(\omega_{0}-\omega\right) T_{2}^{2}}{1+\left(\omega_{0}-\omega\right)^{2} T_{2}^{2}+\omega_{1}^{2} T_{1} T_{2}}
$$

\subsection{Magnetic Susceptibility of DPPH}

This is a dimensionless quantity. We calculate it for DPPH from the formula:

$\frac{M}{B}=\frac{N J U+1) g^{2} \mu_{B}{ }^{2}}{3 K_{B} T}$

$\mathrm{N}$ is the numbers of dipole in unit volume. $\mathrm{N}$ $=21.3811 * 10^{26} \mathrm{~m}^{-3}$.

The total angular momentum quantum number $\mathrm{J}=1 / 2$ (taken for $\mathrm{DPPPH}$ ); $\mathrm{g}=\mathrm{g}_{\mathrm{DPPH}}=2.0038 ; \mathrm{T}=300 \mathrm{~K}$.

After the calculation in S.I. unit

$\frac{M}{B}=\frac{N J U+1) g^{2} \mu_{B}{ }^{2}}{3 K_{B} T}=44.566497 \mathrm{Amp}^{2} /$ Newton

Volume susceptibility in S.I. become,

$X_{0}=\frac{M}{B} \mu_{0}=(44.566497) \frac{A^{2}}{N} * 4 \pi * 10^{-7} \frac{N}{A^{2}}$
$X_{0}=\frac{M}{B} \mu_{0}=(44.566497) * 4 \pi * 10^{-7}$

[Where $\mu_{0}$ is the permeability of the space $\mu_{0}=4 \pi * 10^{-7}$ $\mathrm{NA}^{-2}$ in S.I.]

\subsection{Relaxation Time $\left(T_{1} \& T_{2}\right)$ for DPPH}

$\mathrm{T}_{1}$, the longitudinal relaxation time depends on the spinlattice coupling, and $T_{2}$ the transverse relaxation time depends on spin-spin coupling. For both cases as the coupling becomes stronger the relaxation time becomes longer. And longer relaxation time would produce comparatively low line width $\Delta \mathrm{B}_{\mathrm{PP}}$ (peak to peak distance of the dispersion curve). In case of DPPH line width $\left(\Delta \mathrm{B}_{\mathrm{PP}}\right)$ strongly depends on the solvent in which the substance has been crystallized. The lowest observed value of DPPH crystallized from $\mathrm{CS}_{2}$ is $0.15 \mathrm{mT}$. In general peak to peak line width $\left(\Delta \mathrm{B}_{\mathrm{PP}}\right)$ is reported from $0.15 \mathrm{mT}$ to $0.81 \mathrm{mT}$ and relaxation time $\left(\mathrm{T}_{1} \& \mathrm{~T}_{2}\right)$ are related with the line width $\left(\Delta \mathrm{B}_{\mathrm{PP}}\right)$ by the following relation :- ${ }^{[10]}$

$\Delta B_{p P}{ }^{2}=\frac{4}{a_{P^{2} T_{2}}^{2}}\left(1+B_{1}{ }^{2} T_{1} T_{2}\right) \quad\left[\right.$ Generally $\left.\mathrm{T}_{1}>\mathrm{T}_{2}\right]$

[For DPPH the value of $\mathrm{T}_{2}$ is very close to $\mathrm{T}_{1} \approx T_{2} \approx T_{1}$ ]

[For small value of $B_{1}{ }^{2}$ and very large value of $y^{2}$ we can neglect $B_{1}{ }^{2} \frac{4}{a r^{2}}$ term]

$\Delta B_{p P}^{2} \approx \frac{4}{3 y^{2} T_{2}^{2}}$

$T_{2}^{2}=\frac{4}{3 y^{2} \Delta B_{p p}^{2}}$

$T_{2}=\frac{2}{\sqrt{3}}\left(\frac{1}{y \Delta B_{P P}}\right)$

For simulation purpose we have plotted a graph between $\mathrm{T}_{2}$ vs. $\Delta \mathrm{B}_{\mathrm{PP}}(0.15 \mathrm{mT}$ to $0.81 \mathrm{mT})$ in fig: 34 .

For simulation we have taken $\Delta \mathrm{B}_{\mathrm{PP}}=0.1732050809 \mathrm{mT}$. So, $\mathrm{T}_{1}=\mathrm{T}_{2}=0.378360954 * 10^{-7} \mathrm{sec}$.

\subsection{Values Used In Simulation}

$\mathrm{N}=21.38111 * 10^{26}$ number $/ \mathrm{m}^{3}$

$\mathrm{J}=1 / 2$

$\mathrm{\gamma}=0.17619859 * 10^{12}$ radian $\sec ^{-1} \mathrm{~T}^{-1}$

$\mathrm{g}=$ an isotropic $\mathrm{g}$ value reported in the literature for DPPH is $2.0036 \pm 0.0002[10]$

$\mathrm{K}_{\mathrm{B}}=1.3806488 * 10^{-23} \mathrm{~J} / \mathrm{K}$

$\mathrm{T}=300 \mathrm{~K}$

$\mu_{\mathrm{B}}=9.2849 * 10^{-24} \mathrm{JT}^{-1}$

$\mathrm{B}_{0}=5.0316^{*} 10^{-4} \mathrm{~T}, 5.0316^{*} 10^{-2} \mathrm{~T}, 5.0316^{*} 10^{-1} \mathrm{~T}, 5.0316 \mathrm{~T}$

$\mathrm{T}_{1}=\mathrm{T}_{2}=0.378360954 * 10^{-7} \mathrm{sec}$.

$X_{0}=4 \pi * 10^{-7} * 44.566497$ 


\section{EXPLANATION OF ALL THE SIMULATED OUTPUT GRAPH IN MATLAB}

(In this simulation we have used equation (1), (2) for power absorption and dispersion respectively.)

\subsection{Simulation After Neglecting $\omega_{1}^{2} T_{1} T_{2}$ Terms}

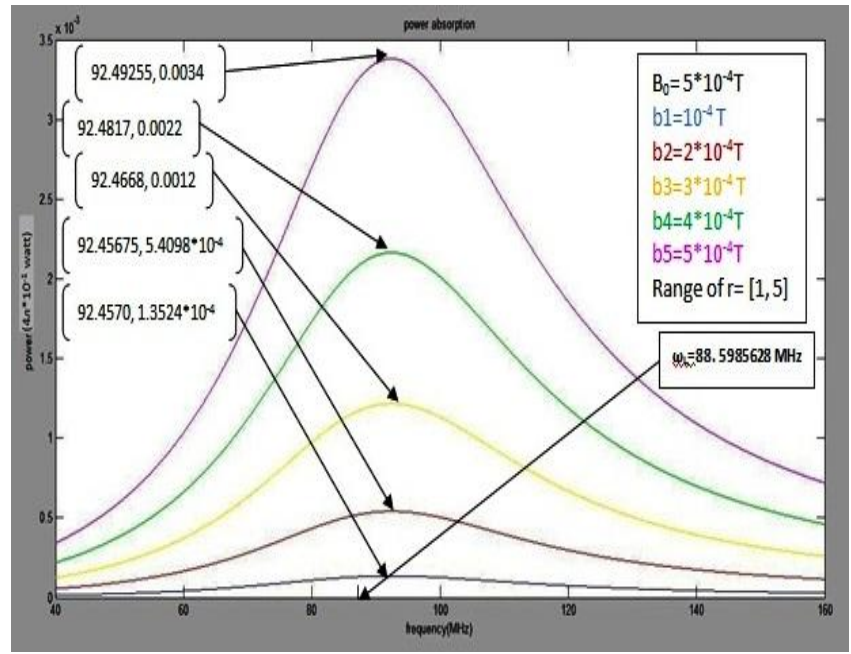

Fig 3:- b1=10.^-4; b2=2*10.^-4; b3=3*10.^-4; b4=4*10.^-4; $\mathrm{b} 5=5 * 10{ }^{\wedge}-4$. (b1, b2, b3, b4, and b5 are different magnetic field).In this graph for each curve magnetic field along $\mathrm{z}$ axis $\left(\mathrm{B}_{0}\right)$ is constant but $\mathrm{rf}$ field is time varying along $\mathrm{x}$ axis also $\left(B_{1}\right)$ is different for each and every curve. We have observed that with the increase in the magnetic field the maximum power absorption point is also increases. There is also a slight shift of the median of maximal's from Larmor frequency (ex: for b1 $=10^{-4} \mathrm{~T}$ median of maximal's is at $89.16265 \mathrm{MHz}$ but $\left.\omega_{\mathrm{L}}=88.5985628 \mathrm{MHz}\right)$.

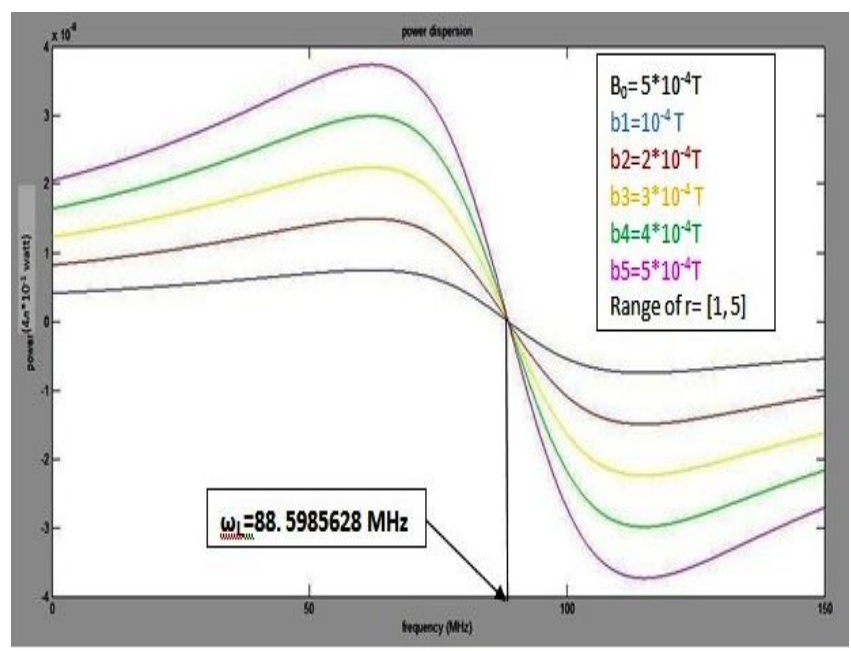

Fig 4:- b1=10.^-4; b2=2*10.^-4; b3=3*10.^-4; b4=4*10.^_ $4 ; \mathrm{b} 5=5^{*} 10 \wedge^{\wedge}-4$. (b1, b2, b3, b4, and b5 are different magnetic field). Frequency is in $\mathrm{MHz}$ range (radio frequency). In this graph the condition is same as fig 3 .

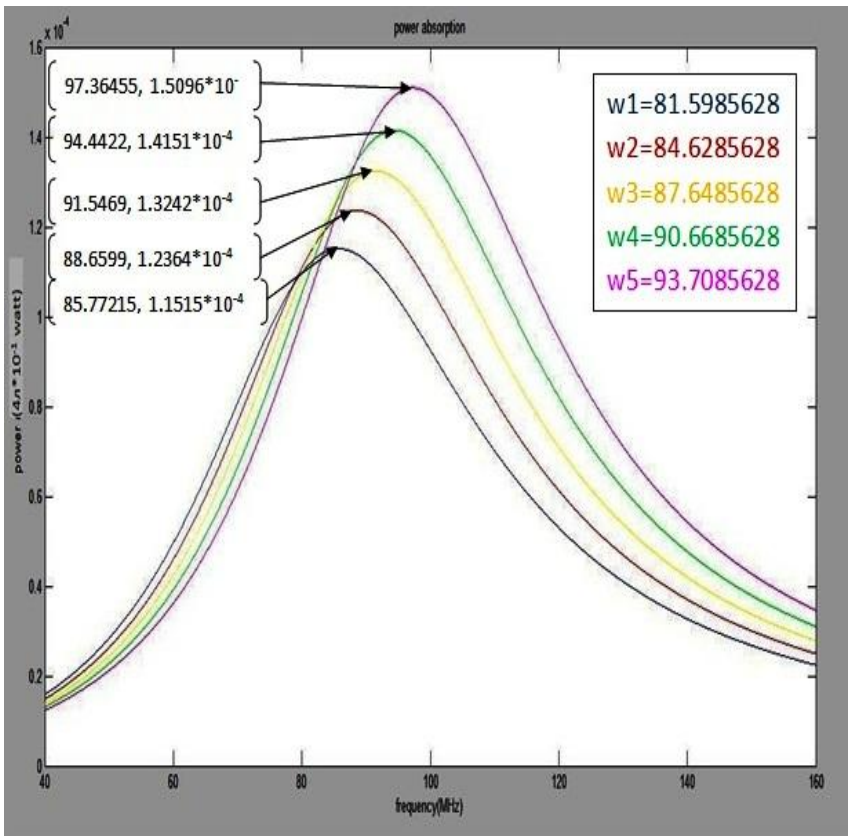

Fig 5:- $w 1=81.5985628 ; w 2=84.6285628 ; w 3=87.6485628$; $\mathrm{w} 4=90.6685628$; w5 $=93.7085628$ (w1, w2, w3, w4, and w5 are Larmor frequency in $\mathrm{MHz}$ range). In this graph for each curve magnetic field $\left(\mathrm{B}_{1}=10^{-4}\right.$.) is constant but $\left(\mathrm{B}_{0}\right)$ is different for each and every curve. For the variation in $\left(\mathrm{B}_{0}\right)$ we have taken different values of frequency $\left(\omega_{0}\right)$ is denoted by w1, w2.etc.By changing the value of Larmor frequency we can sifts the point of the power absorption. In this case the frequency is in the range of $\mathrm{MHz}$, so the bandwidth is large and accuracy is less. Also the maximum points are increasing w.r.t. $\omega_{0}$.

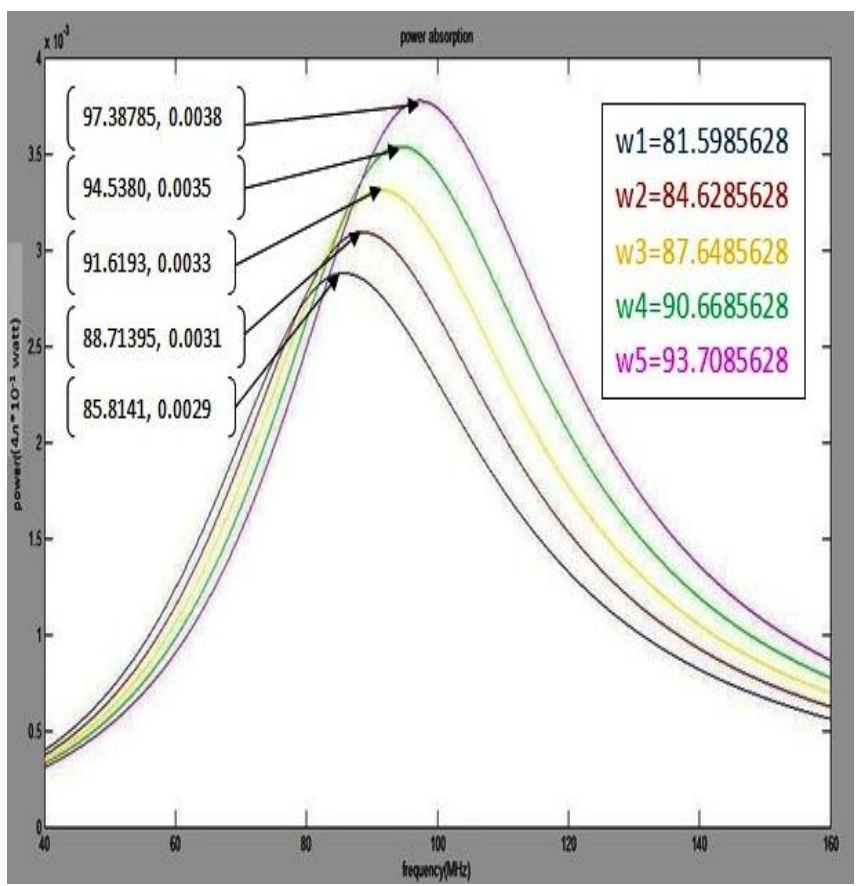

Fig 6:- $w 1=81.5985628 ; w 2=84.6285628$; $33=87.6485628$; $\mathrm{w} 4=90.6685628$; w5 $=93.7085628$ (w1, w2, w3, w4, and w5 are Larmor frequency in $\mathrm{MHz}$ range). In this graph for each curve magnetic field $\left(\mathrm{B}_{1}=5^{*} 10^{-4}\right.$ Tesla) is constant. 


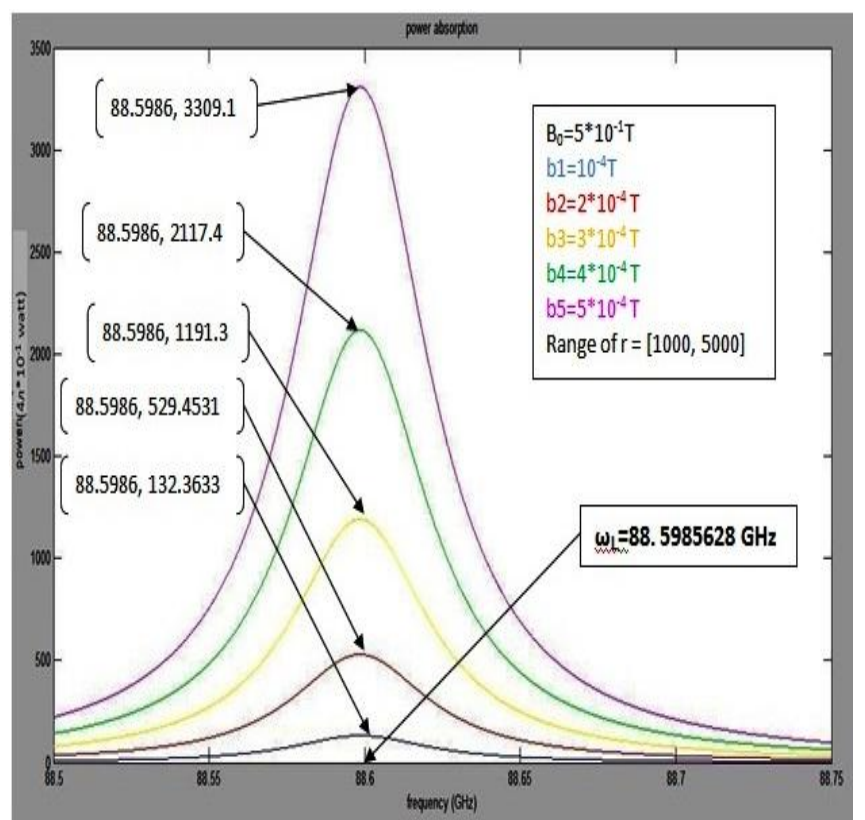

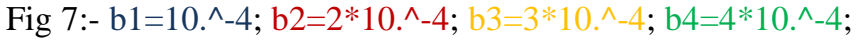
$\mathrm{b} 5=5^{*} 10 .^{\wedge}-4$. (b1, b2, b3, b4, and b5 are different magnetic field).In this graph for each curve magnetic field along $\mathrm{Z}$ axis $\left(B_{0}\right)$ is constant but micro wave field is time varying along $\mathrm{x}$ axis also $\left(\mathrm{B}_{1}\right)$ is different for each and every curve. We have observed that with the increase in the magnetic field the maximum power absorption point is also increases. For the micro wave frequency the shift of maximal's are not observed up to four decimal place but we will observe if we able to calculate the values for more than four decimal place.

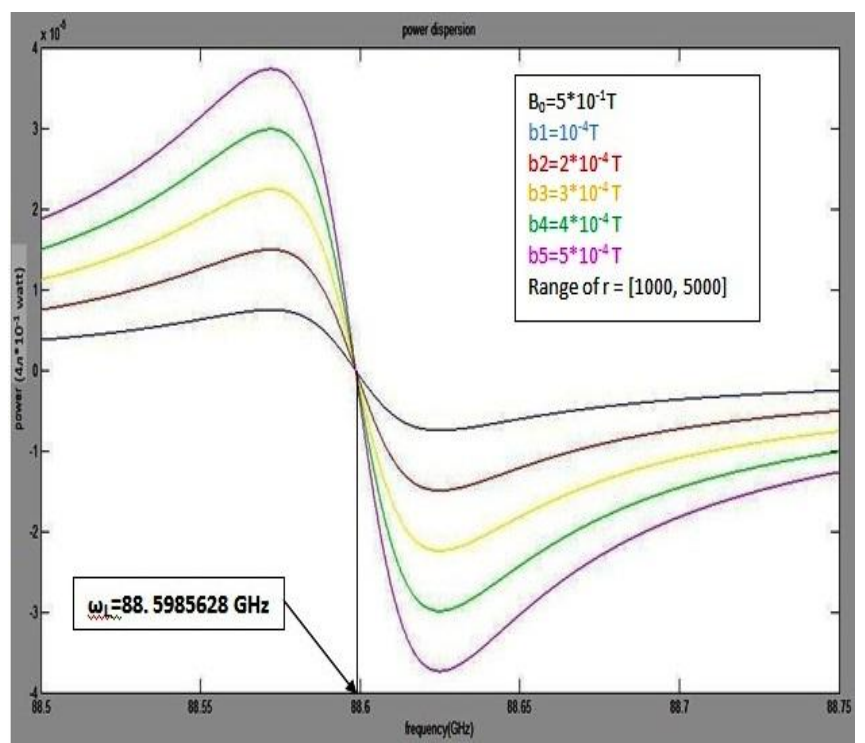

Fig 8:- b1=10.^-4; b2=2*10.^-4; b3=3*10.^-4; b4=4*10.^$4 ; \mathrm{b} 5=5 * 10 \wedge^{\wedge}-4$. (b1, b2, b3, b4, and b5 are different magnetic field). Frequency is in $\mathrm{GHz}$ range (microwave frequency). In this graph for each curve magnetic field along $\mathrm{Z}$ axis $\left(\mathrm{B}_{0}\right)$ is constant but micro wave field is time varying along $\mathrm{x}$ axis also $\left(\mathrm{B}_{1}\right)$ is different for each and every curve. The accuracy in the case of microwave frequency is very high.

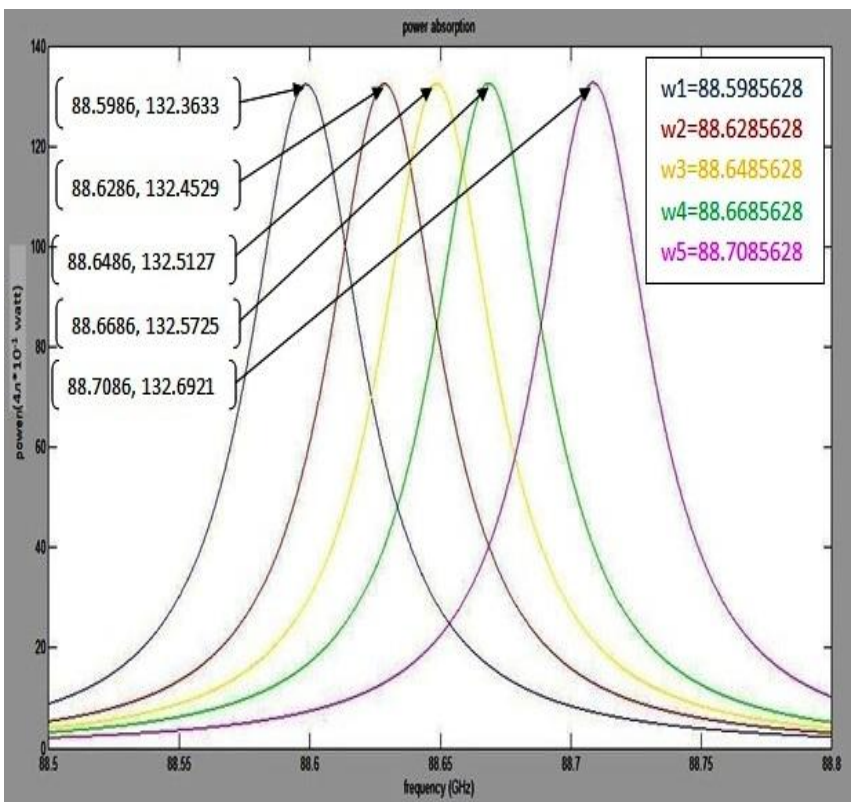

Fig 9:- $w 1=88.5985628 ; w 2=88.6285628 ; w 3=88.6485628$; $\mathrm{w} 4=88.6685628 ; \mathrm{w} 5=88.7085628$ (w1, w2, w3, w4, and w5 are Larmor frequency in $\mathrm{GHz}$ range).In this graph for each curve magnetic field $\left(B_{1}\right)$ is constant but micro wave field is time varying along $\mathrm{x}$ axis also $\left(\mathrm{B}_{0}\right)$ is different for each and every curve. For the variation in $\left(B_{0}\right)$ we have taken different values of frequency $\left(\omega_{0}\right)$. By changing the value of Larmor frequency we can sifts the point of the power absorption. In this case the frequency is in the range of $\mathrm{GHz}$, so the bandwidth is very small and accuracy is very high. For $\mathrm{w} 1=88.5985628 \mathrm{GHz}$ the median of the maximal's is sifts at $88.5987 \mathrm{GHz}$.

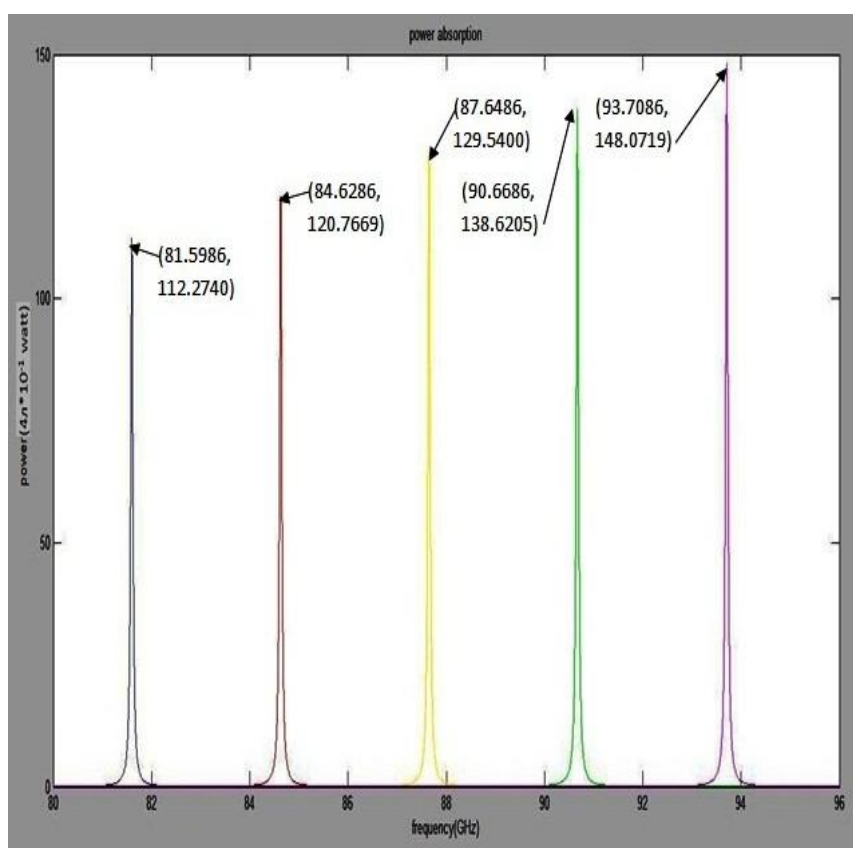

Fig 10:- $\quad w 1=81.5985628 ; \quad w 2=84.6285628$; w3 =87.6485628; w4=90.6685628; w5=93.7085628 (w1, w2, w3, w4, and w5 are Larmor frequency in GHz range). In this graph conditions are same as fig 17 . Here changes of the absorption maxima with the Larmor frequency are prominent. For $w 3=87.6485628 \mathrm{GHz}$ the median of the maximal's is sifts at $87.6488 \mathrm{GHz}$. 


\subsection{Simulation Without Neglecting $\omega_{1}{ }^{2} T_{1} T_{2}$ Terms}

(Study the dependency of steady state power absorption and dispersion output on the ratio $r$ )

We have also simulated power absorption and power dispersion curve without neglecting the value of $\omega_{1}{ }^{2} \mathrm{~T}_{1} \mathrm{~T}_{2}$. Results of simulations are given below. The value of $\omega_{1}$ is taken in the $\mathrm{MHz}$ range in all simulations

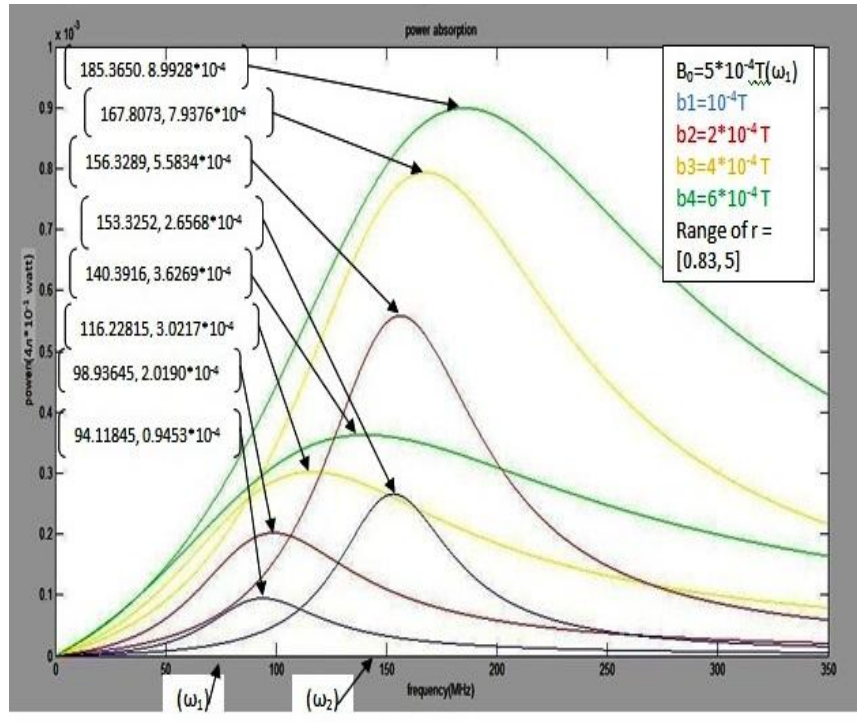

Fig 11:- b1=10.^-4; b2=2*10.^-4; b3=4*10.^-4; b4=6*10.^4. (b1, b2, b3, b4, and b5 are different magnetic field).In this graph for each curve magnetic field along $\mathrm{z}$ axis $\left(\mathrm{B}_{0}\right)$ is constant but $\mathrm{rf}$ field is time varying along $\mathrm{x}$ axis also $\left(\mathrm{B}_{1}\right)$ is different for each and every curve. (In this graph $B_{0}$ is constant even though the maximum power absorption point is at different frequency for the curves having different value of magnitude of magnetic field $\mathrm{B}_{1}$.).In fig 11,12 we have taken two values of $\omega_{0}\left(\omega_{1}=88.5985628 \mathrm{MHz}\right.$ and $\omega_{2}$ $=150 \mathrm{MHz}$ as indicated in graphs)

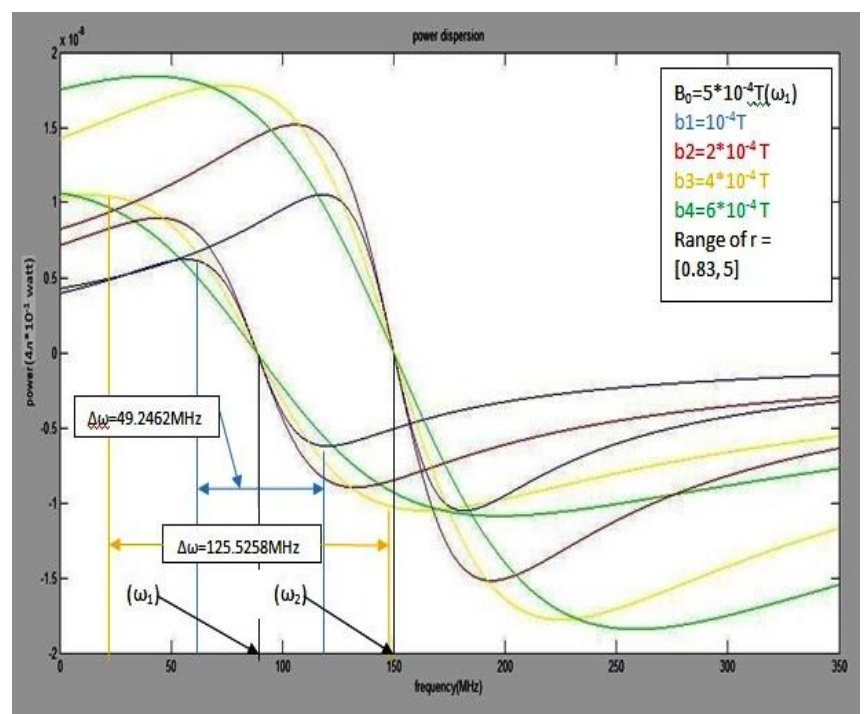

Fig 12:- b1=10.^-4; b2=2*10.^-4; b3=4*10.^-4; b4=6*10.^4. (b1, b2, b3, b4, and b5 are different magnetic field). (In this graph we can see that as the magnitude of the magnetic field $\mathrm{B}_{1}$ increases bandwidth is also increases.)

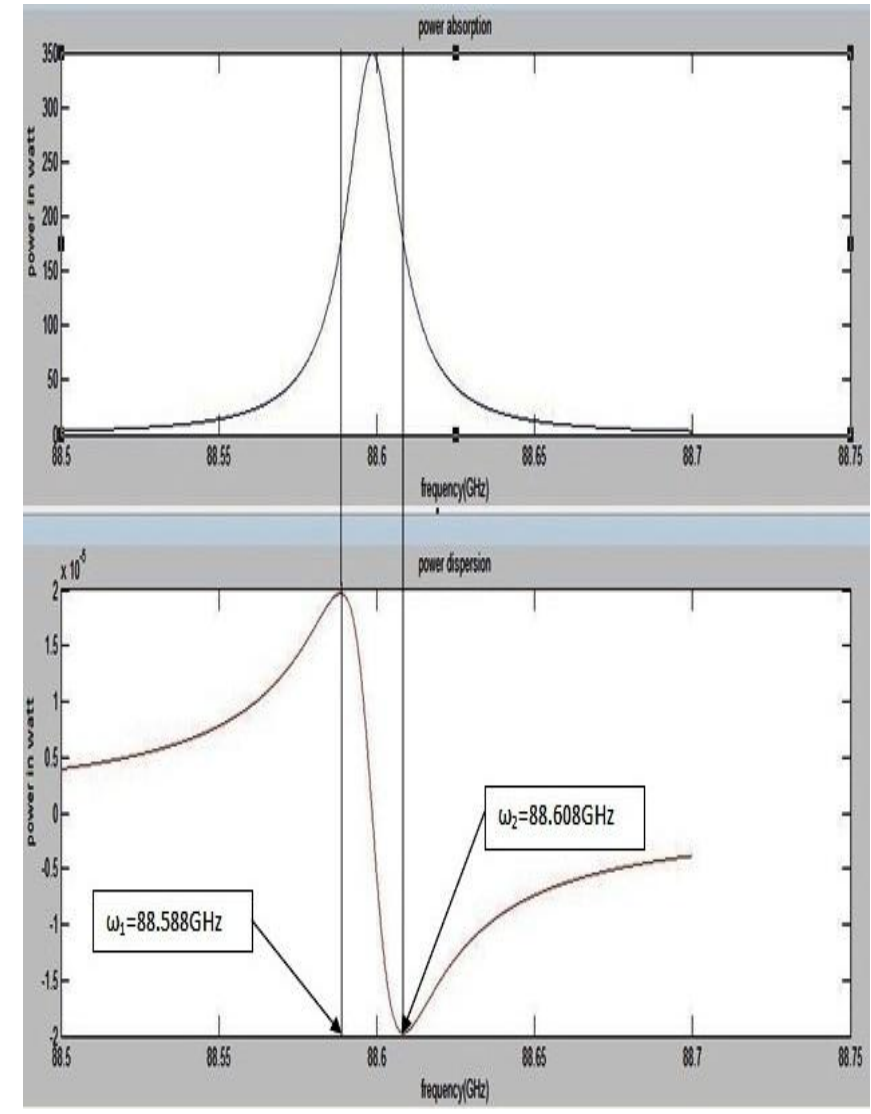

Fig 13:-After calculation we have got $\Delta \omega=\left|\omega_{2}-\omega_{1}\right|=2 * 10^{7} \approx \frac{2}{T_{2}}$

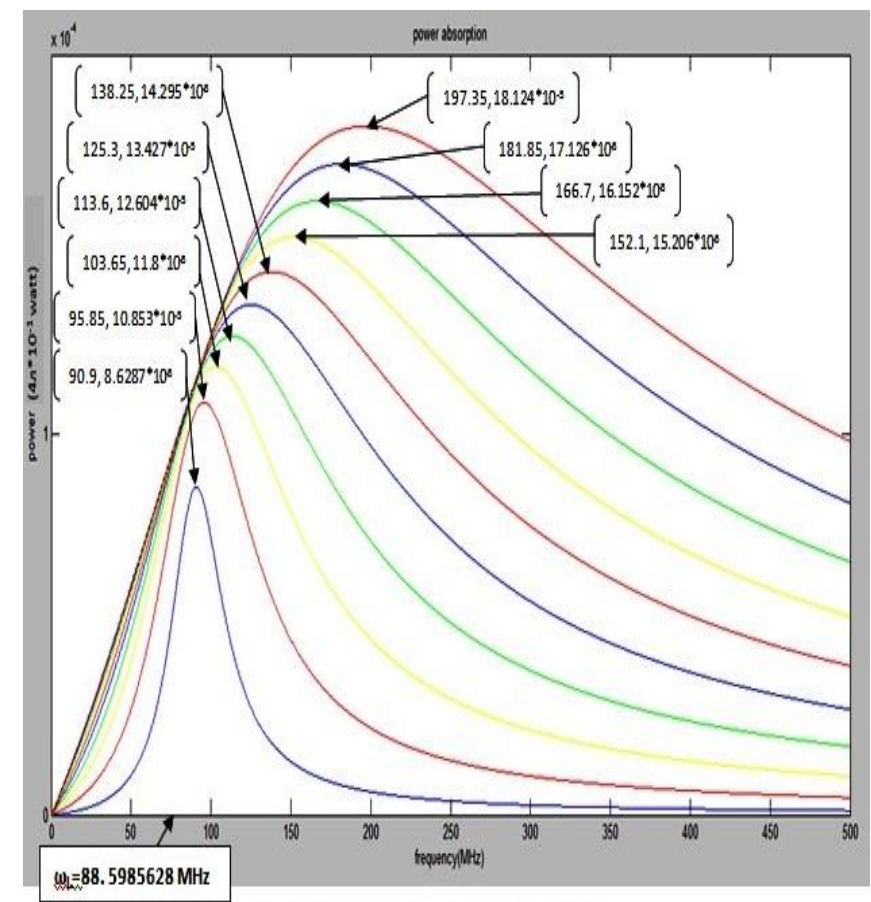

Fig 14 


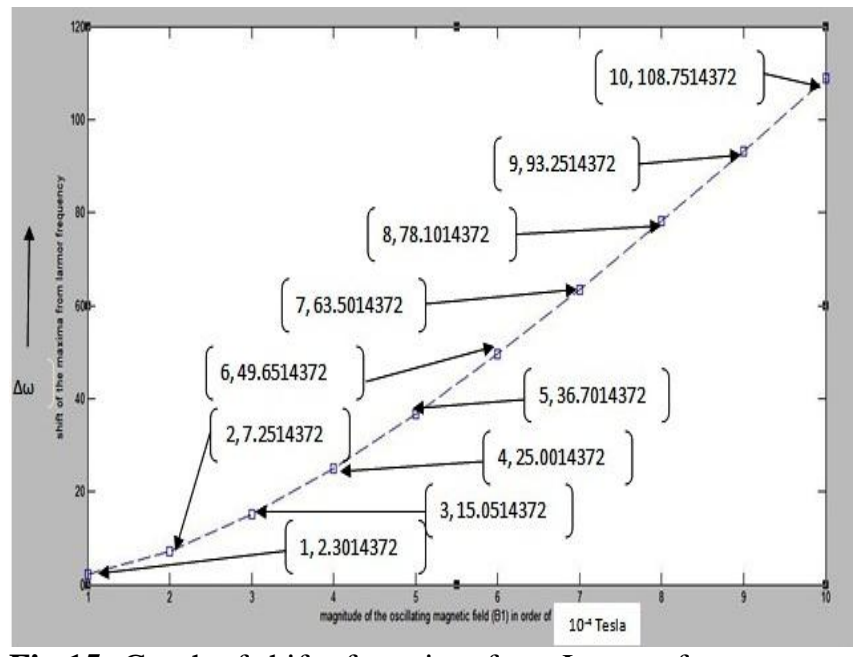

Fig 15: Graph of shift of maxima from Larmor frequency vs magnitude of oscillating magnetic field. In this graph we can see that the shift is non linear.

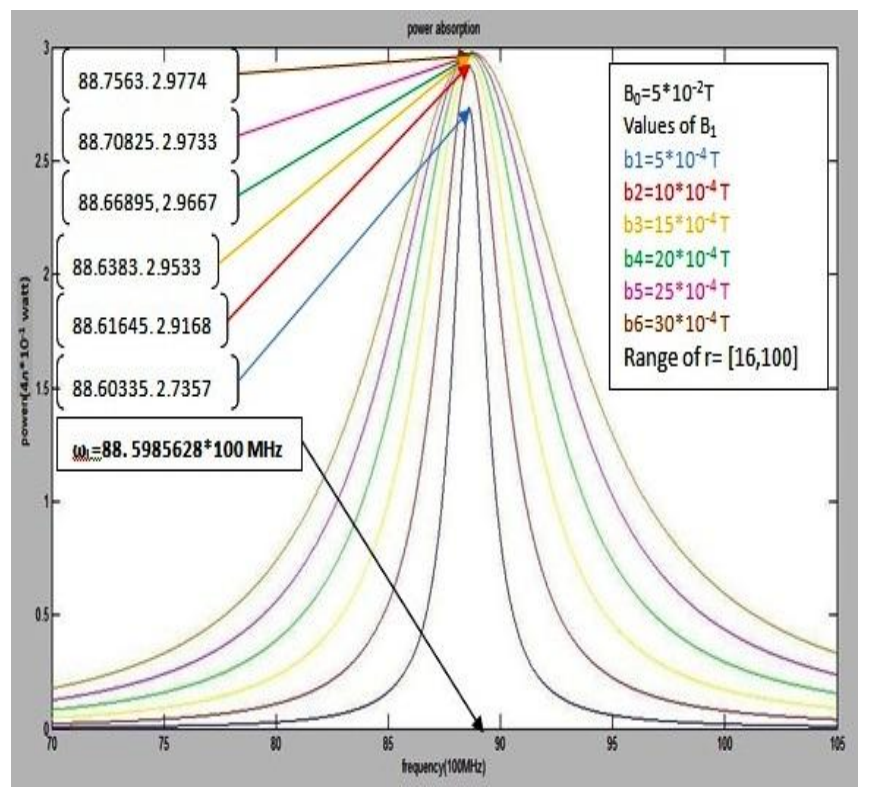

Fig 16

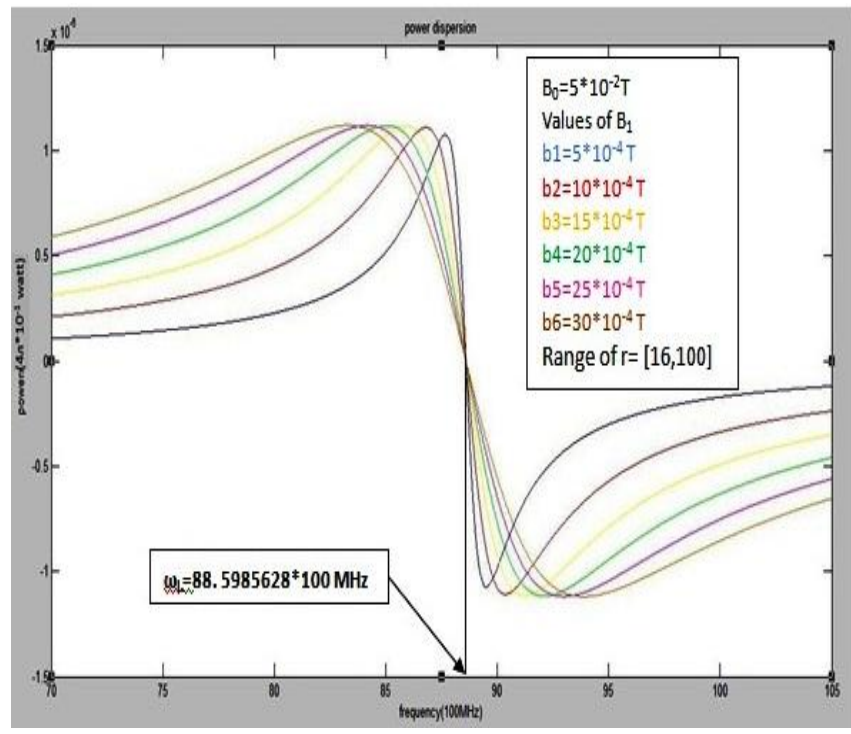

Fig 17

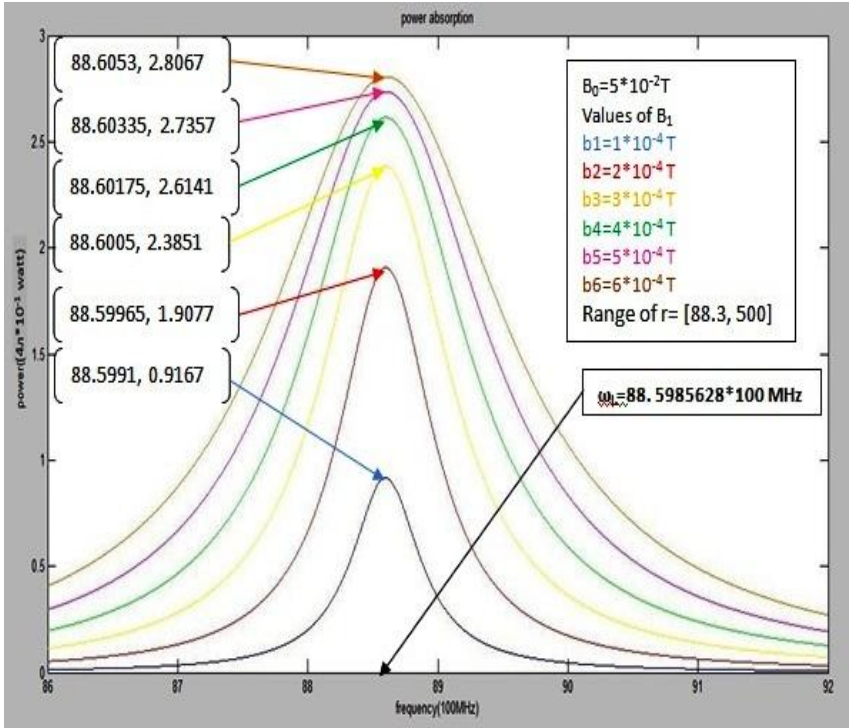

Fig 18

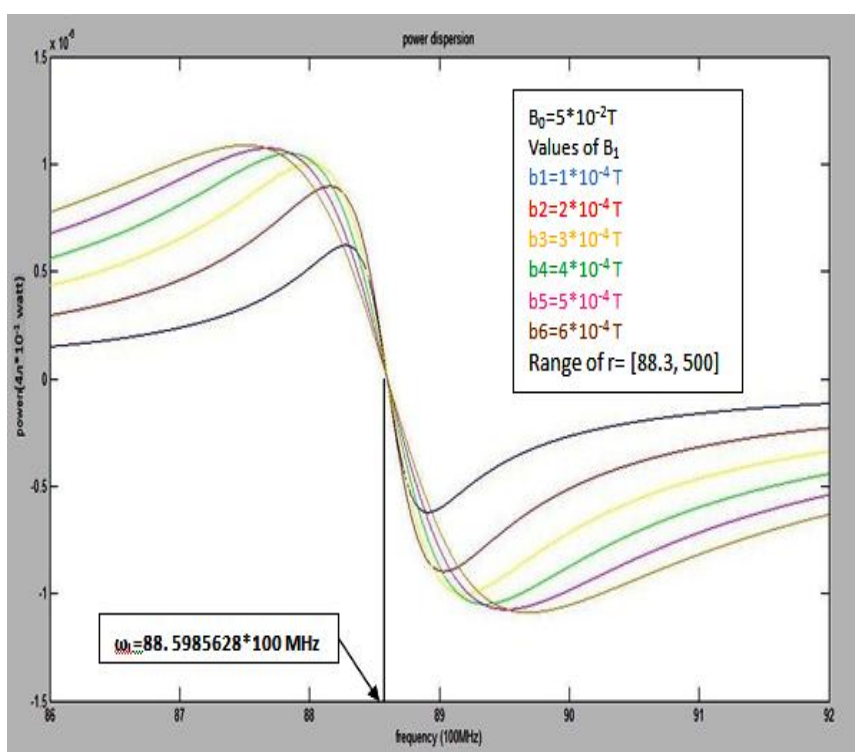

Fig 19

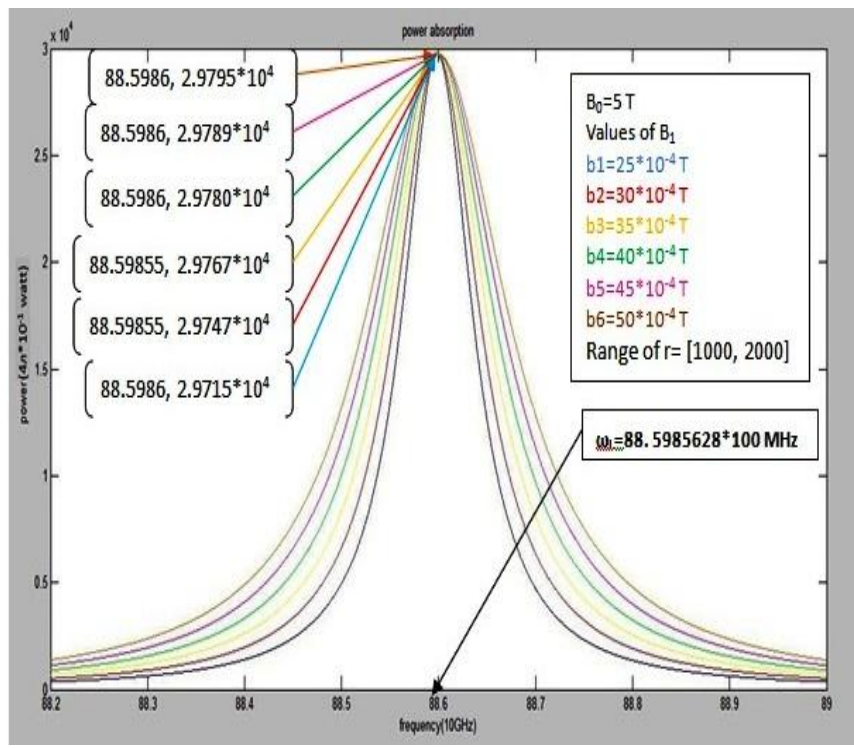

Fig 20 


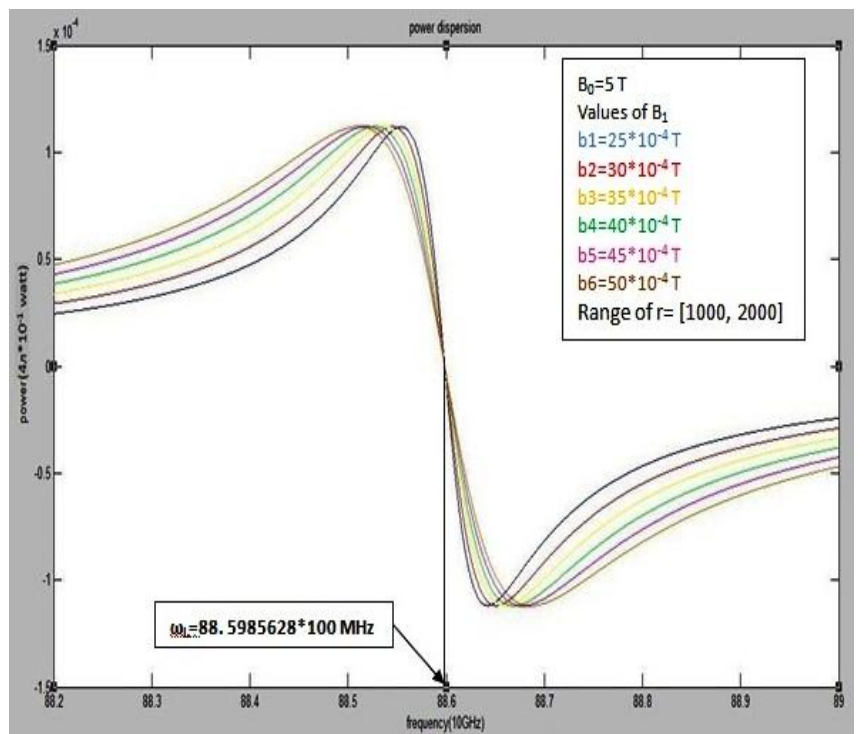

Fig 21

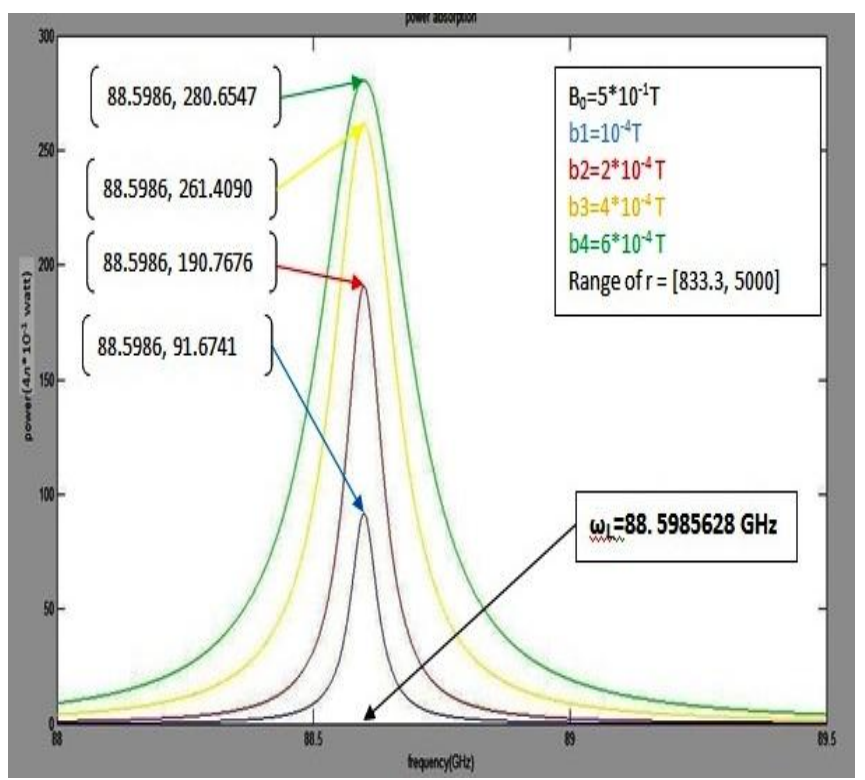

Fig 22

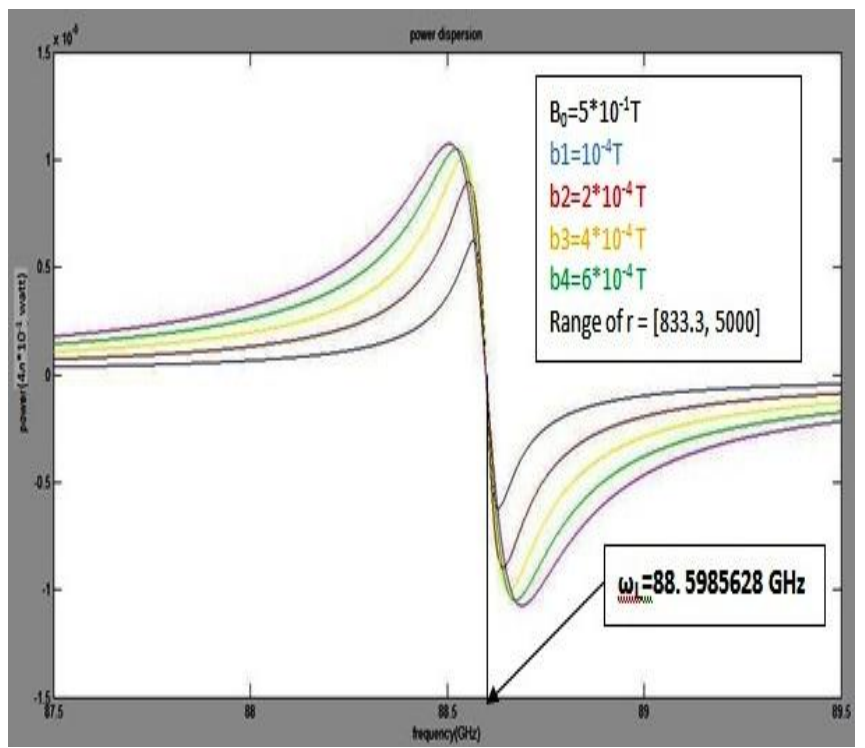

Fig 23

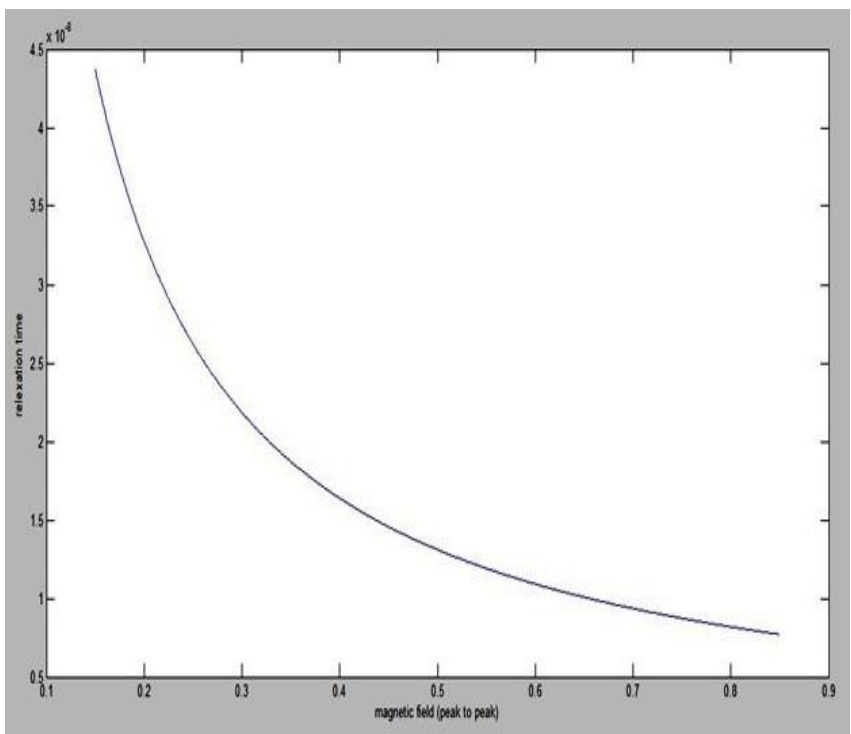

Fig 24:- graph for the relaxation time vs magnetic field peak to peak $\left(\Delta \mathrm{B}_{\mathrm{PP}}\right)$.

\section{CONCLUSIONS}

In the first part of the simulation after ignoring the $\omega_{1}{ }^{2} T_{1} T_{2}$ terms we have observed the dependency of the power maximum on the amplitude of the oscillating e.m. field $B_{1}$ (for fixed $\left(\omega_{\mathrm{L}}\right)$ Larmor frequency) and on $\omega_{\mathrm{L}}$ (for fixed $\mathrm{B}_{1}$ ). Also a clear shift $(\Delta \omega)$ of the power maxima $\left(\mathrm{P}_{\max }\right)$ from $\omega_{\mathrm{L}}$ is observed.

In our second part of the simulation we have considered the $\omega_{1}^{2} T_{1} T_{2}$ term and we have observed as the ratio $\mathrm{r}$ increases the output i.e. shift $(\Delta \omega)$ and the change in $\Delta \mathrm{B}_{\mathrm{PP}}$ with $\mathrm{B}_{1}$ decreases and converges to the case of neglecting $\omega_{1}{ }^{2} T_{1} T_{2}$ terms. We also observe the shift $(\Delta \omega)$ follows some non linear relationship with $\mathrm{B}_{1}$.

It would be very interesting to simulate the response of the system if applied micro wave field is not simply cosine function but a different periodic function of time and carry some information (as an amplitude modulated micro wave field or frequency modulated micro wave field.).

\section{REFERENCES}

[1]. https://en.wikipedia.org/wiki/Bloch_equations.

[2].

https://en.wikipedia.org/wiki/Electron_paramagnetic_resona nce

[3]. https://en.wikipedia.org/wiki/Zeeman_effect

[4]. https://en.wikipedia.org/wiki/Land\%C3\%A9_g-factor

[5]. https://en.wikipedia.org/wiki/Gyromagnetic_ratio

[6]. https://en.wikipedia.org/wiki/Larmor_precession

[7].http://www.unistuttgart.de/gkmr/lectures/lectures_WS_0

203/ magnetisation_blochequ.PDF

[8]. Book Eighth edition introduction to Solid State Physics by CHARLES KITTEL

[9]. http://www.its.caltech.edu/ derose/labs/exp6.html

[10]. N. D. Yordanov, Appl. Magn. Reson. 10 (1996) 339350 . 
[11]. J. A.Weil, J. R. Bolton, J. E.Wertz, Electron Paramagnetic Resonance, John Wiley and Sons, Inc., New York, 1994.

[12]. R. KIRMSE and J. STACH, ESR-Spektroskopie Anwendungen in der Chemie. Akademie-Verlag, Berlin, 1985.

[13]. A. ABRAGAM, Principles of Nuclear Magnetism. Oxford University Press, Oxford,1961.

[14]. R. ERNST, G. BODENHAUSEN, and A. WOKAUN, Principles of Nuclear Magnetic Resonance in One and Two Dimensions. Clarendon Press, Oxford, 1987.

[15]. A.ASTASHKIN and A. SCHWEIGER, Chem. Phys. Lett. 174, 595 (1990).

[16]. S. STOLL, G. JESCHKE, M.WILLER, and A. SCHWEIGER, J. Magn. Reson. 130, 86(1998).

[17]. J. HORNAK and J. FREED, J. Magn. Reson. 67, 501 (1986).

[18]. G. JESCHKE. New Concepts in Solid-State Pulse Electron Spin Resonance. PhDthesis, ETH Z"urich, No. 11873, 1996.

[19]. C. POOLE and H. FARACH, Relaxation in Magnetic Resonance. Academic Press,New York, 1971.

[20]. K. STANDLEY and R. VAUGHAN, Electron Spin Relaxation Phenomena in Solids.Hilger, London, 1969.

[21]. R.Brandle; G.Kruger and W.Muller warmuth; Z.Naturforsch 25,1,((1970)

[22]. Measurement of the longitudinal relaxation time by continues-wave;non linear electron spin resonance spectroscopy J.Magn. reson. 131; 86-91

[23]. Electrons spin resonance absorption in metals.i. Experimental G.Fcher A.F.Kip, physics review 1955 APS.

[24]. Line space of electron paramagnetic resonance signals producd by powder glasses and viscous liquid, Fritz Kurt Kneibhul, the journal of chemical physics 1960.

[25]. Physics of Atoms and Molecules; B.H.Bransden \& C.J.Joacchain (Prearson publication)

\section{BIOGRAPHIES}

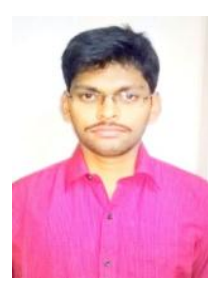

Mr.Amit kumar is Pursing B.Tech Fourth year in Electrical Engineering from Abacus Institute of Engineering and Managament (A.I.E.M.) Natungram, Mogra, Dist. Hooghly W.B., INDIA, Affiliated to West Bengal University of Technology (W.B.U.T.). braj.amit777@gmail.com

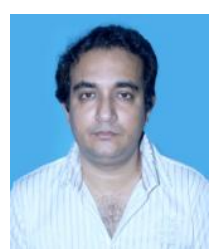

Rajib Chakraborty Assistant professor of physics in the department of physics of A.I.E.M. (Affiliated Tech college of West Bengal University of Technology). Completed B.Sc (Physics hons) from IGNOU and M.Sc physics from Manipal University (Sikkim). Also qualified in CSIR-UGC-NET in physical Sc. Have Research interests mainly on Quantum information Science, Quantum Entanglement and it's influence on quantum Game theory. (rajib_30@yahoo.co.in) 